\title{
Interfacially polymerized thin-film composite polyamide membrane: positron annihilation spectroscopic study, characterization and pervaporation performance
}

\author{
Se-Tsung Kao ${ }^{1,2}$, Shu-Hsien Huang ${ }^{3}$, Der-Jang Liaw ${ }^{1,4}$, Wei-Chi Chao ${ }^{1}$, Chien-Chieh $\mathrm{Hu}^{5}$, Chi-Lan $\mathrm{Li}^{5}$, \\ Da-Ming Wang ${ }^{1,6}$, Kueir-Rarn Lee ${ }^{1}$ and Juin-Yih Lai ${ }^{1}$
}

To improve the pervaporation performance of polyamide membrane, thin-film composite (TFC) polyamide membranes were prepared through the interfacial polymerization between $m$-phenylenediamine (MPDA) or 1,3-phenylenediamine-4-sulfonic acid (MPDASA) and trimesoyl chloride (TMC) on the surface of the modified asymmetric polyacrylonitrile (mPAN) membrane and applied in the pervaporation separation of 70 weight \% aqueous isopropanol solutions at $25{ }^{\circ} \mathrm{C}$. The variations in the free volume and the thickness of the active polyamide layer of composite membrane were obtained by positron annihilation spectroscopy (PAS) experiments, in which a variable monoenergy slow-positron beam was used. FTIR-ATR spectroscopy, XPS, scanning electron microscopy, AFM and water contact angle measurements were applied to analyze chemical structures, surface elemental compositions, morphologies, surface roughness and hydrophilicity of the active polyamide layer of composite membrane. From the result of PAS experiments, the $S$ parameter (corresponding to the free volume size and amount) and the thickness of the active polyamide MPDASA-TMC/mPAN layer were found to be lower than those of the active MPDA-TMC/mPAN layer. In the aqueous isopropanol solution dehydration, the MPDASA-TMC/mPAN membrane exhibited a higher permeation rate than but maintained the same water concentration in the permeate as did the MPDA-TMC/mPAN membrane. This is in good agreement with the analysis by PAS.

Polymer Journal (2010) 42, 242-248; doi:10.1038/pj.2009.334; published online 13 January 2010

Keywords: interfacial polymerization; PAS; pervaporation; polyamide; TFC membrane

\section{INTRODUCTION}

Polyamides have been studied as suitable membrane materials because of their high thermal stability, excellent mechanical strength and high resistance to organic solvents. Alcohol dehydration is one of the important areas in pervaporation separation processes. In pervaporation separation processes, polyamides show high selectivity in alcohol dehydration for a wide range of water concentrations. ${ }^{1-5}$ Their high selectivity stems from the concept of high diffusion selectivity. This is because they have low free volume/low mobility and the size of water molecules is smaller than that of alcohol. However, they show low permeation rate because of their very low free volume and low water solubility. To increase the permeation rate of polyamide membranes without sacrificing selectivity, the membrane morphology must be converted from a dense thick film into an asymmetric or composite morphology. Composite membranes have several advantages over single-material asymmetric membranes. Composite membranes are characterized by a top separation layer formed onto a chemically different asymmetric porous substrate. The benefits of these independent polymeric layers can be combined to obtain the desired membrane performance. Interfacial polymerization is an effective technique to prepare a composite membrane with a selective thin layer. Besides being thin and dense, a more hydrophilic selective layer is favorable to further improve the performance of a membrane for alcohol dehydration.

Interfacial polymerization is based on a polymerization reaction that forms a polymer film at the interface between two immiscible phases (aqueous and organic), each of which has a highly reactive monomer dissolved in it. The polymer film formed at the interface

\footnotetext{
${ }^{1}$ Department of Chemical Engineering, R\&D Center for Membrane Technology, Chung Yuan University, Chung Li, Taiwan; ${ }^{2}$ Institute of Polymer Science and Engineering, National Taiwan University, Taipei, Taiwan; ${ }^{3}$ Department of Chemical and Materials Engineering, National Ilan University, I-Lan, Taiwan; ${ }^{4}$ Department of Chemical Engineering, National Taiwan University of Science and Technology, Taipei, Taiwan; ${ }^{5}$ Department of Chemical and Material Engineering, Nanya Institute of Technology, Chung-Li, Taiwan and ${ }^{6}$ Department of Chemical Engineering, National Taiwan University, Taipei, Taiwan

Correspondence: Professor K-R Lee, Department of Chemical Engineering, Chung Yuan University, No. 200, Chung Pei Rd., Chung Li 32023, Taiwan.

E-mail: krlee@cycu.edu.tw or

Professor S-H Huang, Department of Chemical and Materials Engineering, National Ilan University, No. 1, Sec. 1, Shen-Lung Rd., I-Lan 26047, Taiwan.

E-mail: huangsh@niu.edu.tw
}

Received 12 August 2009; revised 20 November 2009; accepted 25 November 2009; published online 13 January 2010 
usually grows from the aqueous phase toward the organic phase. This concept of polymer film growth at the interface has been proved by Morgan. ${ }^{6}$ The advantages of interfacial polymerization are the following: rapid reaction rates under ambient conditions, the absence of a requirement for stoichiometric amounts of reactants and a low requirement for reactant purity. Thus, the interfacial polymerization technique is an adequate method for the preparation of composite membranes with an ultra-thin active polyamide layer. Studies on interfacially polymerized thin-film composite (TFC) membranes are usually about their application in reverse osmosis ${ }^{7-11}$ or nanofiltration, ${ }^{12-16}$ but few reports on their application in pervaporation are available. ${ }^{17-19}$ Therefore, in this study, to improve the pervaporation performance of polyamide membranes, TFC polyamide membranes were prepared through the interfacial polymerization reaction between amines ( $m$-phenylenediamine (MPDA) or 1,3-phenylenediamine-4sulfonic acid (MPDASA) (sulfonated MPDA)) and acyl chloride (trimesoyl chloride (TMC)) on the surface of a modified asymmetric polyacrylonitrile (mPAN) membrane. These TFC polyamide membranes were then applied in the pervaporation separation of aqueous isopropanol solution at $25^{\circ} \mathrm{C}$ The polyamide layer's characteristics such as morphology, surface roughness, hydrophilicity, degree of crosslinking, free volume and thickness were correlated with the pervaporation performance. It was especially a difficult task to estimate the free volume in the thin polyamide layer on the modified PAN membrane support. Recently, many studies on the physical properties of polymeric materials, such as free volume, have been investigated with a potential physical technique-PAS. ${ }^{20-23}$ However, there are few studies on polymeric TFC pervaporation membranes investigated with PAS. ${ }^{18-19,24-25}$ In this regard, to probe the variation in the free volume in the active polyamide layer, the TFC polyamide membrane was examined by conducting PAS experiments, in which a variable monoenergy slow-positron beam was used.

\section{EXPERIMENTAL PROCEDURE}

\section{Materials}

PAN polymer, supplied by Tong-Hua Synthesis Fiber Co. Ltd (Hsinchu, Taiwan), was used as the membrane support in the interfacially polymerized TFC membrane. Reagent grade $N$-methyl-2-pyrrolidone (NMP) was the solvent used in preparing the casting solution of the PAN polymer. Sodium hydroxide, used in the hydrolysis of the PAN membrane support, was purchased from SHOWA Chemical (Tokyo, Japan). MPDA was purchased from Aldrich (Steinheim, Germany). MPDASA (sulfonated MPDA) and TMC were purchased from TCI (Tokyo, Japan). Amine monomers (MPDA and MPDASA) and acyl chloride (TMC) monomer were used in the preparation of the active polyamide layers through interfacial polymerization. Distilled water was used as the solvent in aqueous solutions, and toluene was used as the organic solvent. The chemical structures of MPDA, MPDASA and TMC are shown in Figure 1.

\section{Preparation of mPAN porous membrane support}

The preparation procedure for the mPAN porous membrane support used in this study was described in our previous study. ${ }^{26}$ Flat porous membrane<smiles>Nc1cccc(N)c1</smiles><smiles>O=C(Cl)c1cc(C(=O)Cl)cc(C(=O)Cl)c1</smiles>

Figure 1 Chemical structures of monomers used in preparing active polyamide layers. (a) MPDA, (b) MPDASA, (c) TMC. support was prepared by casting the PAN-NMP solution onto nonwoven polyester fabrics. In the preparation process, an NMP solution containing 15 weight $\%$ PAN polymers was cast onto a nonwoven polyester substrate with the use of a casting knife with a $200-\mu \mathrm{m}$ gap to fabricate the flat porous PAN membrane support. The cast membrane was precipitated in a water bath. The resulting asymmetric PAN porous membrane support was washed in water several times for more than 1 day to remove the remnant of the NMP solvent.

The modified PAN membrane was prepared by immersing the PAN porous membrane support in a $2 \mathrm{M} \mathrm{NaOH}$ solution at $50^{\circ} \mathrm{C}$ to improve the hydrophilicity of the PAN membrane and to facilitate the spread of the aqueous amine solution over its surface. The partial $-\mathrm{CN}$ groups of PAN can be converted into $-\mathrm{COOH}$ or $-\mathrm{CONH}_{2}$ groups after hydrolysis with $\mathrm{NaOH}$ solution. ${ }^{12,27}$ The resulting modified PAN membrane was washed in a water bath for several hours and then stored in another water bath before use for the interfacial polymerization.

\section{Preparation of TFC polyamide membrane}

The active polyamide skin layer was polymerized by the interfacial polymerization technique. The mPAN membrane was first immersed in a 0.1 weight $\%$ aqueous amine solution for $5 \mathrm{~s}$. Then, the excess amount of aqueous amine solution on the surface of the mPAN membrane was removed using a rubber rod. The mPAN membrane soaked with the aqueous amine solution was contacted with a toluene solution containing 0.05 weight $\%$ acyl chloride (TMC) for $10 \mathrm{~s}$ to carry out the process of interfacial polymerization. Finally, the resulting TFC polyamide membrane was washed in methanol and then dried at atmospheric temperature.

\section{Characterization}

FTIR-ATR (Perkin Elmer Spectrum One, Perkin Elmer Ltd, Beaconsfield, UK) spectroscopy was used to examine the chemical structures of the membranes. Scanning electron microscopy (SEM) (HITACHI S-3000N, Hitachi, Tokyo, Japan) and AFM (Digital Instruments, DI-NS3a, Santa Barbara, CA, USA) were used to observe the morphologies and the surface roughness of the TFC polyamide membranes, respectively. To understand the surface hydrophilicity of the TFC polyamide membrane, the water contact angle was estimated with the use of automatic interfacial tensiometer (FACE Mode 1 PD-VP, Kyowa Interface Science Co., Ltd., Saitama, Japan). The chemical surface characterization of the TFC polyamide membranes was carried out by using XPS (Thermo VGScientific Sigma Probe, Thermo Fisher Scientific, Loughborough, UK).

\section{PAS}

To estimate the variation in the free volume in the TFC polyamide membrane, a newly built slow-positron beam with variable monoenergy at the R\&D Center for Membrane Technology, Chung Yuan University, Taiwan, was used for this study to define the mean depth of the membrane between 0 and about $10 \mu \mathrm{m}$ (the mean depth is converted by using an established equation from the positron incident energy from 0 to $30 \mathrm{keV}$ ) at atmospheric temperature under a vacuum of $\sim 10^{-8}$ torr to measure the Doppler-broadened energy spectrum (DBES). This new radioisotope beam uses $50 \mathrm{mCi}$ of ${ }^{22} \mathrm{Na}$ as the positron source. The DBES spectra were measured using a high-purity Ge detector at a counting rate of $\sim 2000$ c.p.s. The total number of counts for each DBES spectrum was 1.0 million. DBES is based on the measurement of the width of the annihilation gamma photon line, centered at $511 \mathrm{keV}$, by a high-purity Ge detector. The $S$ parameter defined by the ratio of the central part of the annihilation spectrum and the total spectrum reflects positron annihilation with low momentum valence electrons. To estimate the thickness of the active polyamide layer, the S parameter data from DBES was fitted in a four-layer model by using the VEPFIT program. ${ }^{28}$ The $S$ parameter from the DBES represents the relative value of the free volume reflected in the depth profile for polymeric systems.

\section{Pervaporation measurement}

The pervaporation performance measurement was performed by the separation of aqueous isopropanol solution through a TFC polyamide membrane. The pervaporation apparatus was illustrated in our previous study. ${ }^{29}$ The effective surface area of the membrane in direct contact with the feed solution was 
$11.64 \mathrm{~cm}^{2}$. The operating temperature (feed solution temperature) was $25^{\circ} \mathrm{C}$. The concentrations of the feed solution and the permeate were measured by gas chromatography (GC; China Chromatography 9800, Taipei, Taiwan). The permeation rate $(P)$ was calculated from the following equation:

$$
P=\frac{W}{A \times t}
$$

where $W$ is the weight of the permeate, $A$ is the effective membrane area, and $t$ is the sampling time.

\section{RESULTS AND DISCUSSION}

\section{Chemical structures}

The FTIR-ATR spectra for PAN membrane, mPAN membrane and TFC polyamide membranes (MPDA-TMC/mPAN and MPDASATMC/mPAN) are shown in Figure 2. Compared with the spectrum for the PAN membrane (Figure 2a), the mPAN membrane has four peaks at wave numbers $3300-3400,1732,1666$ and $1560 \mathrm{~cm}^{-1}$, as shown in Figure $2 \mathrm{~b}$. The first two peaks correspond to $-\mathrm{OH}$ and $\mathrm{C}=\mathrm{O}$ of the carboxyl acid group, respectively. The last two peaks correspond to $\mathrm{C}=\mathrm{O}$ (amide I) and $-\mathrm{NH}$ (amide II) of the acryl amide group, respectively. Such spectra revealed that partial $-\mathrm{CN}$ groups of the PAN membrane were converted to the $-\mathrm{COOH}$ or $-\mathrm{CONH}_{2}$ groups, as a result of PAN hydrolysis in $\mathrm{NaOH}$ solution.

Compared with the spectrum for the mPAN membrane, an increase in the intensity of the peaks at wave numbers 1666 and $1560 \mathrm{~cm}^{-1}$, corresponding to $\mathrm{C}=\mathrm{O}$ (amide $\mathrm{I}$ ) and $\mathrm{N}-\mathrm{H}$ (amide II) of the amide group, respectively, is shown in the spectra for the TFC polyamide membranes (MPDA-TMC/mPAN and MPDASA-TMC/mPAN) (Figures $2 \mathrm{c}$ and $\mathrm{d}$ ). In addition, the spectrum for MPDASA-TMC/ mPAN exhibits two new peaks at 1022 and $1078 \mathrm{~cm}^{-1}$ (showing in the magnified portions in the wave number range $950-1150 \mathrm{~cm}^{-1}$ from Figures $2 \mathrm{c}$ and $\mathrm{d}$ ), which were not indicated in the spectrum for MPDA-TMC/mPAN (Figure 2c). These peaks indicate the characteristic of the aromatic $-\mathrm{SO}_{3} \mathrm{H}$ symmetric and asymmetric stretching vibrations, respectively. ${ }^{30}$ The spectra in Figure 2 confirmed that the TFC membrane's active layer is composed of aromatic polyamide.

\section{SEM observation}

Figure 3 shows the SEM surface images of the mPAN and TFC polyamide membranes. As shown in Figure 3a, the mPAN membrane exhibits a smooth surface with micropores spread throughout. Compared with the mPAN membrane, it was found that the thin active polyamide layers were formed on the mPAN membranes through the interfacial polymerization process, as shown in Figures $3 b$ and c. In Figure $3 \mathrm{~b}$, the polyamide MPDA-TMC on the mPAN membrane is delineated as crooked, wormlike strands connected with each other. Compared with the MPDA-TMC/mPAN membrane, the smoother polyamide MPDASA-TMC having small, well-dispersed nodules on the mPAN membrane is shown in Figure 3c. The smoother surface of the MPDASA-TMC/mPAN membrane could result from the lower polymerization rate of the reaction of MPDASA with TMC because of the steric hindrance effect of $-\mathrm{SO}_{3} \mathrm{H}$ groups in MPDASA. In view of this, the SEM cross-sectional images of the TFC polyamide membranes were also examined. The thickness of the active polyamide layer was not observed clearly.

\section{Surface roughness and hydrophilicity measurement}

The surface roughness and the hydrophilicity of the membrane can affect the pervaporation performance of the membrane. Generally, higher surface roughness (larger surface area) has an opportunity to promote the permeation rate. Higher hydrophilicity is favorable not only to improve the water concentration in the permeate but also the permeation rate. Thus, the surface roughness and the hydrophilicity of the TFC polyamide membranes were investigated.

From the SEM observation, the MPDASA-TMC/mPAN membrane has a smoother surface than the MPDA-TMC/mPAN membrane. Further, the closer surface morphology and roughness of these two TFC polyamide membranes were probed by AFM, as shown in Figure 4 and Table 1. Figure 4 shows AFM images of MPDA-TMC/ mPAN and MPDASA-TMC/mPAN membranes. Compared with the MPDASA-TMC/mPAN membrane, the MPDA-TMC/mPAN membrane has a rougher surface because of the small bumps distributed on it. The surface roughness data from Figure 4 are also shown in Table 1. It indicates that the root mean square roughness $\left(R_{\mathrm{ms}}\right)$, average roughness $\left(R_{\mathrm{a}}\right)$ and maximum roughness $\left(R_{\max }\right)$ for the MPDATMC/mPAN membrane are higher than those for the MPDASATMC/mPAN membrane. These results might be attributed to MPDA having a higher polymerization rate of reaction with TMC than MPDASA.

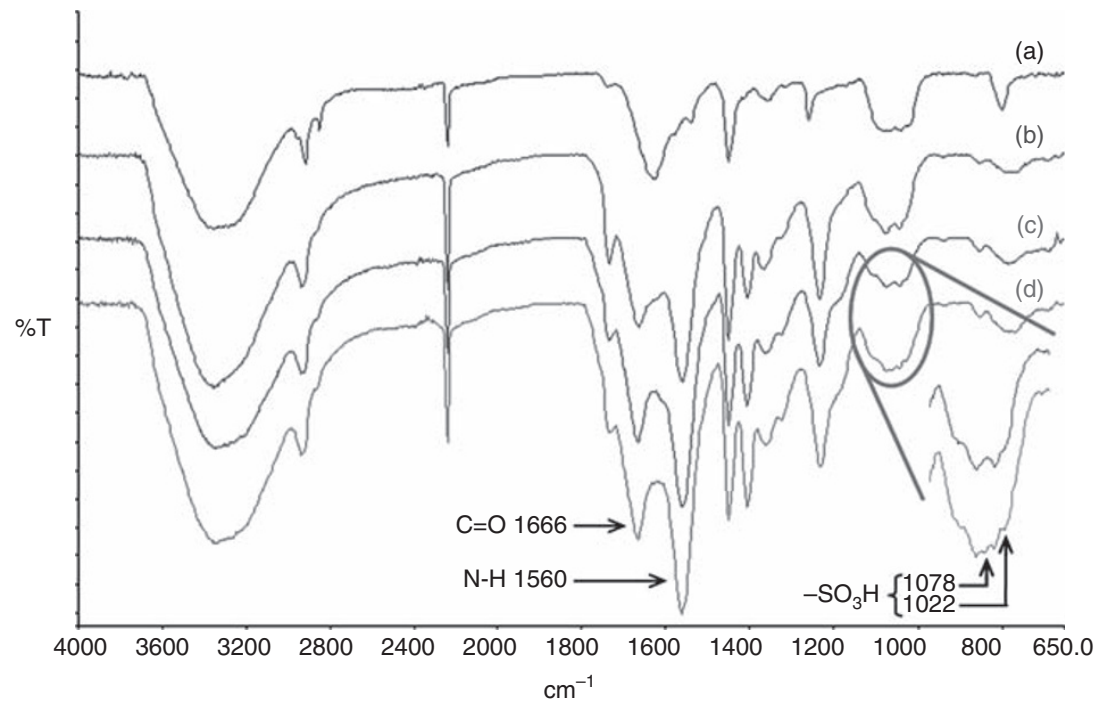

Figure 2 FTIR-ATR spectra for (a) pristine PAN membrane support, (b) modified PAN (mPAN) membrane support (result of PAN treatment in $2 \mathrm{~m}$ NaOH solution at $50^{\circ} \mathrm{C}$ ) and TFC polyamide membranes (c) MPDA-TMC/mPAN and (d) MPDASA-TMC/mPAN. 

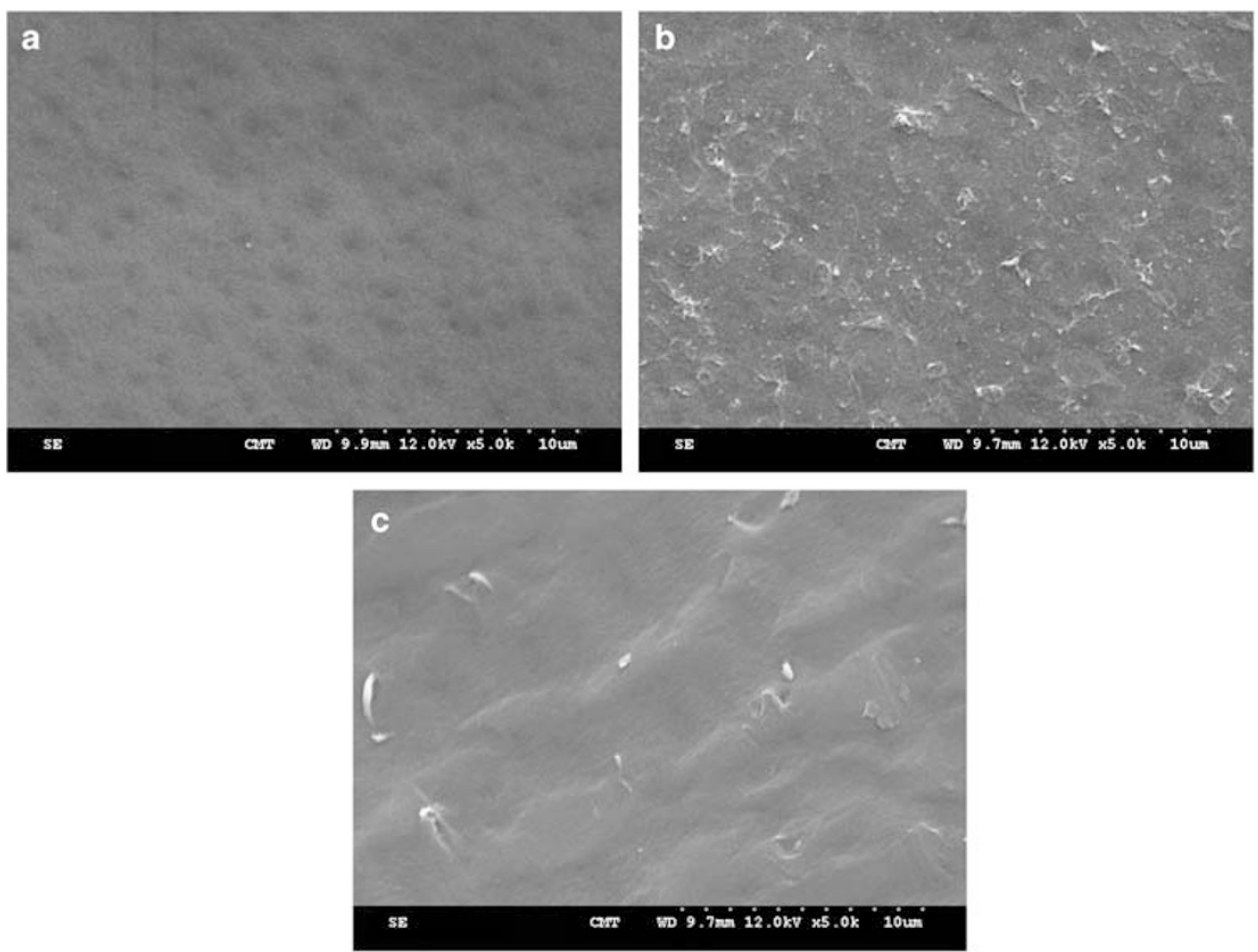

Figure 3 SEM surface images of (a) modified PAN (mPAN) membrane support and TFC polyamide membranes (b) MPDA-TMC/mPAN and (c) MPDASA-TMC/ mPAN.
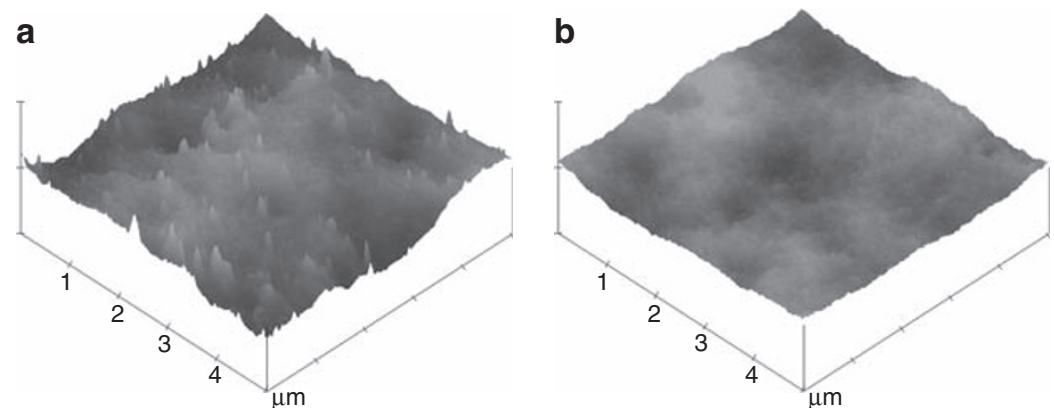

Figure 4 AFM images of TFC polyamide membranes (a) MPDA-TMC/mPAN and (b) MPDASA-TMC/mPAN. (scan size: $5 \times 5 \mu$; ordinate scale (data scale): $200 \mathrm{~nm}$ per interval).

Table 1 Surface roughness and water contact angle values for TFC polyamide membranes

\begin{tabular}{lllll} 
Membranes & $\mathrm{R}_{m s}{ }^{\mathrm{a}}(\mathrm{nm})$ & $\mathrm{R}_{a}{ }^{\mathrm{b}}(\mathrm{nm})$ & $\mathrm{R}_{\max }{ }^{\mathrm{c}}(\mathrm{nm})$ & $\begin{array}{r}\text { Water contact } \\
\text { angle }\left({ }^{\circ}\right)\end{array}$ \\
\hline MPDA-TMC/mPAN & $26.2 \pm 2.0$ & $20.8 \pm 2.1$ & $190.6 \pm 9.7$ & $68.1 \pm 0.7$ \\
MPDASA-TMC/mPAN & $13.2 \pm 1.1$ & $10.4 \pm 0.9$ & $89.3 \pm 8.7$ & $33.5 \pm 4.7$ \\
\hline
\end{tabular}

Abbreviations: mPAN, modified asymmetric polyacrylonitrile; MPDA, $m$-phenylenediamine; MPDASA, 1,3-phenylenediamine-4-sulfonic acid; TFC, thin-film composite; TMC, trimesoyl chloride.

${ }^{\text {a }} R_{\mathrm{ms}}$ : root mean square roughness.

${ }^{\mathrm{b}} R_{\mathrm{a}}$ : average roughness.

${ }^{c} R_{\text {max }}$ : maximum roughness.

Two factors can affect the hydrophilicity of the membrane. One is the hydrophilic groups on the membrane. The membrane having some hydrophilic groups can decrease the water contact angle of the membrane, that is, improve the hydrophilicity of the membrane. Another is the surface roughness of the membrane. The surface roughness of the membrane is generally favorable to increase the hydrophilicity of the membrane whose water contact angle is lower than $90^{\circ} \mathrm{C}$. Therefore, the hydrophilicity of the TFC polyamide membranes was directly estimated using the water contact angle data and the result is shown in Table 1. It was found that the MPDASA-TMC/mPAN membrane has a lower water contact angle than the MPDA-TMC/mPAN membrane. This result indicates that the MPDASA-TMC/mPAN membrane has a higher hydrophilicity because of the hydrophilic $-\mathrm{SO}_{3} \mathrm{H}$ groups in it, although it has a lower surface roughness.

\section{Crosslinking degree estimation}

In the pervaporation separation process, the polymer membrane is easily swollen by the organic feed solution. This result causes a decrease in the selectivity and an increase in the permeation rate. 
The crosslinking structure formed in the polymer membrane can inhibit the swelling effect of the organic feed solution and is beneficial to maintain the pervaporation performance. In this study, the reaction between TMC having three acyl chloride groups and MPDA or MPDASA possessing two amine groups could form the polyamide with the crosslinking structure. Thus, the crosslinking portion in the polyamide thin layer was estimated by the surface elemental composition of the composite membrane, which was obtained from XPS. Table 2 shows the surface elemental composition and the elemental concentration ratio data for the TFC polyamide membranes from the XPS measurement. As shown in Table 2, the MPDASA-TMC/mPAN membrane has a higher $\mathrm{N} / \mathrm{C}$ ratio and a lower $\mathrm{O} / \mathrm{N}$ ratio compared with the MPDA-TMC/mPAN membrane. This result indicates that more polyamide linkage groups (crosslinking portions) formed in the MPDASA-TMC polyamide layer through interfacial polymerization. Figure 5 illustrates the chemical structures of the polyamides (MPDATMC and MPDASA-TMC). As shown in Figure 5, the polyamide structure was divided into two portions, that is, the crosslinking portion $(m)$ and the linear portion $(n)$. The fraction of crosslinking portion $(m)$ of the polyamide layer was estimated from the N/C ratio for the repeating units in the polyamide structure. In the calculation, $m+n=1$ and $\mathrm{N} / \mathrm{C}=(3 m+2 n) /(18 m+15 n)$ from the chemical formulae of the crosslinking portion $(m)$ and the linear portion $(n)$ were used. The result of the calculation is shown in Table 2. It indicates that the MPDASA-TMC polyamide layer has a higher fraction of crosslinking portion than MPDA-TMC. These phenomena might be due to the fact that the polyamide layer formed by means of interfacial polymerization usually grows from the aqueous phase toward the organic phase. As the interface thickness increases, the amounts of aqueous phase monomer that can penetrate decreases. On reaction with TMC, MPDA has a higher polymerization rate than MPDASA. The polyamide is, therefore, formed rapidly. This MPDA-TMC polyamide layer causes the MPDA monomers' penetration to decrease, resulting in some acyl chloride groups of TMC that cannot react with the amine groups of MPDA. After the interfacial polymerization process and on exposure to the atmosphere, the unreacted acyl chloride groups will be hydrolyzed to form the $-\mathrm{COOH}$ groups. Thus, the MPDA-TMC polyamide layer has a lower fraction of crosslinking portion. On the other hand, MPDASA has a lower polymerization rate than MPDA, resulting from the steric hindrance effect of the $-\mathrm{SO}_{3} \mathrm{H}$ group. Hence, the polyamide forms gradually. The MPDASA monomers penetrate more easily through the thinner MPDASA-TMC polyamide layer,

Table 2 Surface elemental composition, elemental concentration ratio and crosslinking portion data for TFC polyamide membranes from XPS measurement

\begin{tabular}{|c|c|c|c|c|c|c|c|c|}
\hline Membranes & $C(\%)$ & $O(\%)$ & $N(\%)$ & $S(\%)$ & $\mathrm{O} / \mathrm{C}$ & $N / C$ & $O / N$ & Crosslinking portion (m) (\%) \\
\hline MPDA-TMC/mPAN & 75.07 & 14.22 & 10.71 & - & 0.19 & 0.14 & 1.33 & 17 \\
\hline MPDASA-TMC/mPAN & 74.71 & 12.61 & 12.27 & 0.41 & 0.17 & 0.16 & 1.03 & 77 \\
\hline
\end{tabular}

Abbreviations: mPAN, modified asymmetric polyacrylonitrile; MPDA, m-phenylenediamine; MPDASA, 1,3-phenylenediamine-4-sulfonic acid; TFC, thin-film composite; TMC, trimesoyl chloride.

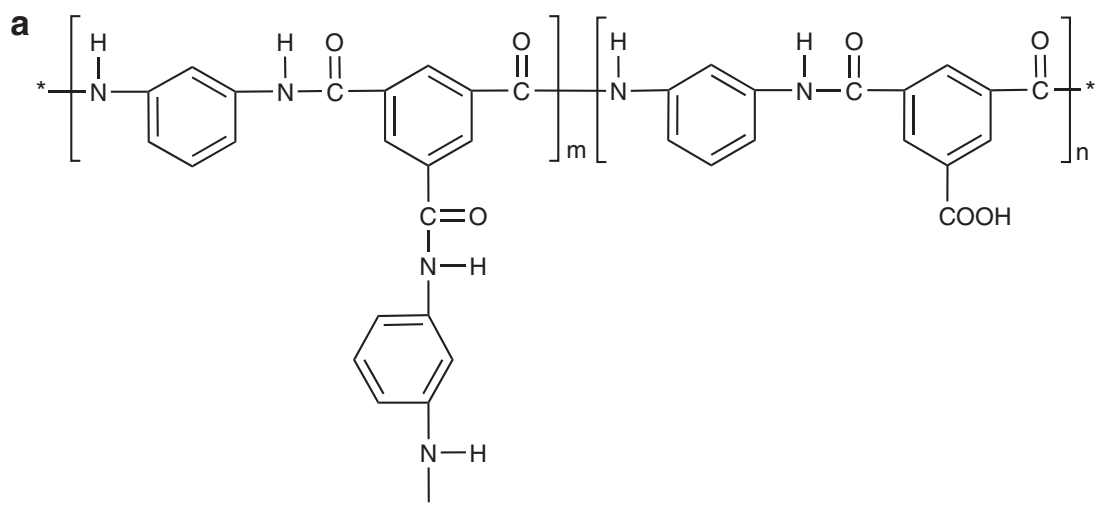

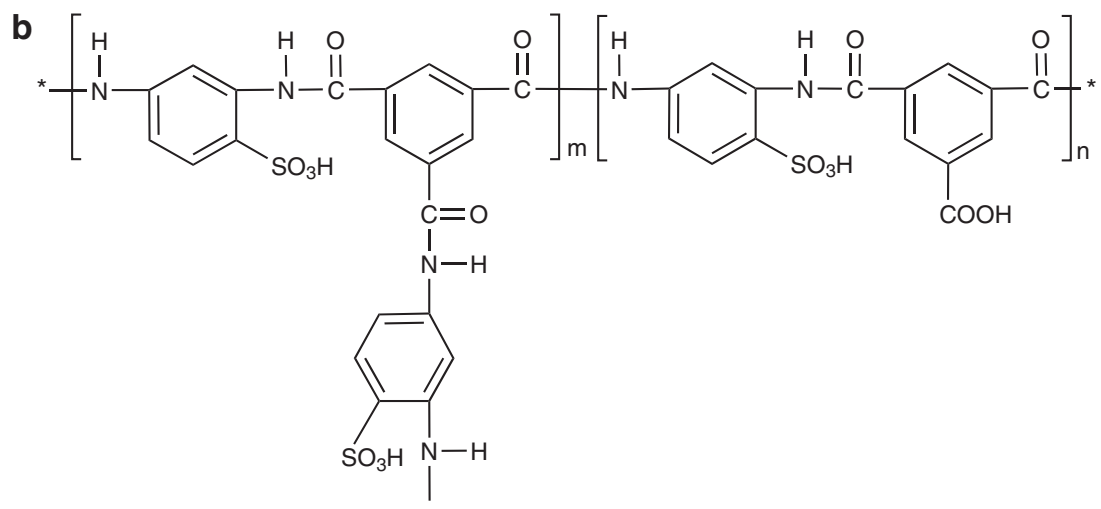

Figure 5 Illustration of chemical structures of polyamides (a) MPDA-TMC and (b) MPDASA-TMC. 
compared with the thicker MPDA-TMC layer. This favors the acyl chloride groups of TMC to react with the amine groups of the MPDASA to a greater extent, resulting in a higher fraction of crosslinking portion.

\section{Free volume and thickness estimations through PAS}

Both the free volume and the thickness of the polymer membrane can affect the performance of the membrane applied in the pervaporation separation process. Larger free volume in the polymer membrane is favorable to improve the permeation rate. The thinner polymer membrane is also beneficial to promote the permeation rate because of the lower resistance to mass transfer.

It is a difficult task to estimate the free volume in the thin polyamide layer on the modified PAN membrane support. In this study, the variation in the free volume in the TFC polyamide membrane was examined by conducting a PAS experiment. PAS experiment with the use of a variable monoenergy slow-positron beam was performed to obtain DBES. In general, DBES can be described by a lineshape $S$ parameter. The $S$ parameter value increases by increasing the relative contribution of low momentum electrons to the positron annihilation in the open volume voids. ${ }^{18-19,24-25}$ DBES can, therefore, be applied to measure the variation in free volume in a composite membrane. The multilayer structure of a composite membrane can be analyzed from the $\mathrm{S}$ parameter variation (free volume variation).

The $S$ parameter, as a function of the positron incident energy from DBES for the TFC polyamide membranes is exhibited in Figure 6. The following observations can be drawn from such an S curve plot: (1) The $S$ parameter near the surface increases rapidly as the positron incident energy increases (at values lower than about $1 \mathrm{keV}$ ). (2) There is a small plateau existing at some distance from the membrane surface. (3) The S parameter jumps to another plateau. (4) The S parameter increases to a maximum as the positron incident energy increases further. (5) After attaining the maximum value, the $S$ parameter decreases as a function of the positron incident energy. From the results of the $S$ curves in Figure 6, the rapid increase in the $S$ parameter near the membrane surface at the positron incident energy value of lower than about $1 \mathrm{keV}$ is a typical phenomenon of positronium annihilation. After its rapid increase, the $\mathrm{S}$ parameter attains two plateaus, increases and then decreases, with an increase in the positron incident energy. This variation in the $S$ parameter, resulting from the variation in the fine-structure characteristic of the free volume, indicates a multilayer structure of the TFC polyamide

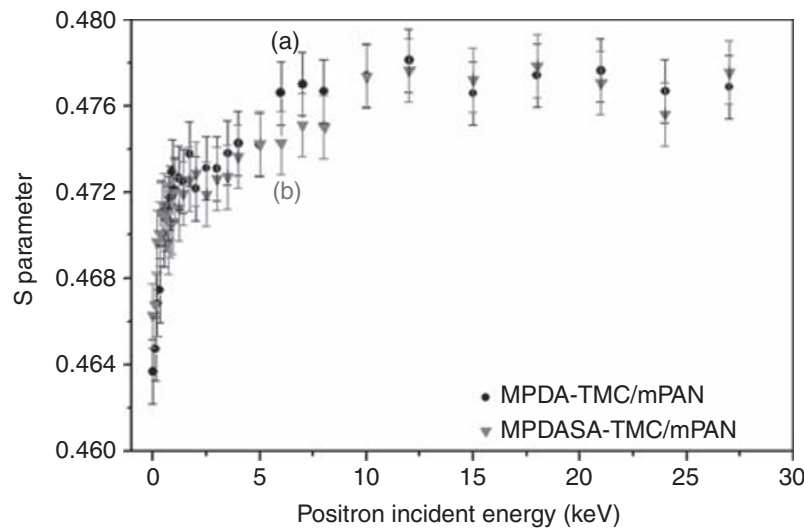

Figure $6 \mathrm{~S}$ parameter as function of positron incident energy for TFC polyamide membranes (a) MPDA-TMC/mPAN and (b) MPDASA-TMC/mPAN. membrane, which is based on the positron annihilation characteristic differences between the layers.

To further explore the variation in the $\mathrm{S}$ parameter along the depth of the composite membrane, the $S$ parameter as a function of the positron incident energy was fitted by the VEPFIT program analysis. We tried to fit three- and four-layer models from the program analysis to examine the layer boundary for each $S$ curve for MPDA-TMC/ mPAN and MPDASA-TMC/mPAN membranes. We found that the $S$ curve fit using a three-layer model showed poor resolution. However, the $S$ curve fit using a four-layer model showed good $\chi^{2}$-values $(<2.5)$ and gave stable results and reasonable error bars. As a result of the VEPFIT analysis in a four-layer model, the TFC polyamide membrane was divided into four layers. For the MPDASA-TMC/mPAN membrane, for example, the order of the multilayer structure is as follows. The first two plateaus pertain to the active MPDASA-TMC polyamide layer $(1.0-3.0 \mathrm{keV})$ and the dense skin layer of the mPAN membrane $(3.0-8.0 \mathrm{keV})$. The next increase in the $\mathrm{S}$ parameter represents a transition layer from the dense skin layer to the porous support layer of the mPAN membrane $(8.0-18 \mathrm{keV})$. Finally, the decrease in the $S$ parameter represents the porous support layer of the mPAN membrane $(>18 \mathrm{keV})$. As shown in Figure 6, the first plateau (the active polyamide layer) of the MPDA-TMC/mPAN membrane is higher than that of the MPDASA-TMC/mPAN membrane. This implies that the free volume in the former membrane is higher than that of the latter membrane.

The effective selective layer of the TFC membrane is the active polyamide layer. Table 3 tabulates the $S$ parameters corresponding to the free volume and the thickness of the active polyamide layers estimated from the VEPFIT program analysis. On the basis of the data in Table 3, the S parameter (corresponding to the free volume size and amount) and the thickness of the active MPDASA-TMC layer are lower than those of MPDA-TMC. The free volume in the active MPDASA-TMC layer is smaller because of the higher fraction of the crosslinking portion in the layer, as shown in Table 2. The thinner active MPDASA-TMC layer might be the cause for the MPDASA to have a lower polymerization rate of reaction with TMC because of the steric hindrance effect of the $-\mathrm{SO}_{3} \mathrm{H}$ groups in MPDASA.

\section{Pervaporation performance}

Table 4 indicates the pervaporation performance of TFC polyamide membranes for separating 70 weight $\%$ aqueous isopropanol solutions at $25^{\circ} \mathrm{C}$. As shown in Table 4, the MPDASA-TMC/mPAN membrane has a higher permeation rate compared with the MPDA-TMC/mPAN membrane. The water concentration in the permeate for the MPDASA-TMC/mPAN membrane is the same as that for the MPDA-TMC/mPAN membrane. The higher permeation rate for the MPDASA-TMC/mPAN membrane is due to its being thinner and its having higher hydrophilicity, despite the smaller free volume in

Table $3 \mathrm{~S}$ parameter $(S 1)$ and thickness $(L 1)$ of polyamide layers of composite membranes from multilayer analysis of $S$ data using VEPFIT program of four-layer model

\begin{tabular}{lcc}
\hline Polyamide layers & $\mathrm{S}^{\mathrm{a}}$ & $\mathrm{L1}^{\mathrm{a}}(\mathrm{nm})$ \\
\hline MPDA-TMC & $0.4728 \pm 0.0004$ & $152.9 \pm 44.3$ \\
MPDASA-TMC & $0.4724 \pm 0.0118$ & $127.4 \pm 67.8$
\end{tabular}

Abbreviations: MPDA, m-phenylenediamine. MPDASA, 1,3-phenylenediamine-4-sulfonic acid TMC, trimesoyl chloride.

aS1 and L1 are, respectively, S parameter and thickness of first layer deduced from S data aS1 and L1 are, respectively, S parameter and thickness of first
fitting in four-layer model based on VEPFIT program analysis. 
Table 4 Pervaporation performance of TFC polyamide membranes for separating 70 weight \% aqueous isopropanol solutions at $25^{\circ} \mathrm{C}$

\begin{tabular}{lcc}
\hline Membranes & Permeation rate $\left(\mathrm{g} \mathrm{m}^{-2} \mathrm{~h}^{-1}\right)$ & $\begin{array}{c}\text { Water concentration. } \\
\text { in permeate (weight \%) }\end{array}$ \\
\hline MPDA-TMC/mPAN & $1522 \pm 102$ & $99.7 \pm 0.1$ \\
MPDASA-TMC/mPAN & $1669 \pm 26$ & $99.7 \pm 0.3$ \\
\hline
\end{tabular}

Abbreviations: mPAN, modified asymmetric polyacrylonitrile; MPDA, $m$-phenylenediamine; MPDASA, 1,3-phenylenediamine-4-sulfonic acid; TFC, thin-film composite; TMC, trimesoyl chloride.

the active MPDASA-TMC layer. The MPDASA-TMC/mPAN membrane maintains the water concentration in the permeate. This result is due to the higher hydrophilicity of, and the higher crosslinking degree for, the active MPDASA-TMC layer, which provides higher ability to inhibit the swelling effect of the aqueous isopropanol feed solution.

\section{CONCLUSIONS}

The TFC polyamide membranes were successfully prepared by the interfacial polymerization of MPDASA or MPDA and TMC and were applied in the dehydration of aqueous isopropanol solution. In summing up the abovementioned experimental results, the following several conclusions were obtained:

1. As a result of PAS experiment, the active MPDASA-TMC layer of the composite membrane had a lower S parameter (corresponding to the free volume size and amount) and was thinner compared with the active MPDA-TMC layer.

2. From the analyses of SEM, AFM, XPS and water contact angle measurements, the MPDASA-TMC/mPAN membrane had a lower surface roughness, a higher crosslinking degree for the active layer and a higher hydrophilicity than the MPDA-TMC/ mPAN membrane.

3. In the dehydration of aqueous isopropanol solution, the MPDASA-TMC/mPAN membrane showed a higher permeation rate than but maintained the same water concentration in the permeate as did the MPDA-TMC/mPAN membrane. A permeation rate of $1669 \mathrm{~g} \mathrm{~m}^{-2} \mathrm{~h}^{-1}$ and a water concentration in the permeate higher than 99 weight \% were obtained with the MPDASA-TMC/mPAN membrane in the dehydration of a 70 weight $\%$ aqueous isopropanol solution at $25^{\circ} \mathrm{C}$.

4. The pervaporation performance results corresponded closely with those of PAS and characterization for the TFC polyamide membranes.

\section{ACKNOWLEDGEMENTS}

We sincerely thank the Toward Sustainable Green Technology project in Chung Yuan University, Taiwan, under grant CYCU-97-CR-CE, the Ministry of Economic Affairs, the Ministry of Education Affairs and the National Science Council of Taiwan for financially supporting this work.

1 Akihiro, Y., Tyagi, R. K., Fouda, A. \& Matsuura, T. Effect of evaporation time on the pervaporation characteristics through homogeneous aromatic polyamide membranes. II. Pervaporation performances for ethanol/water mixture. J. Appl. Polym. Sci. 60, 743-748 (1996)

2 Shieh, J. J. \& Huang, R. Y. M. Preparation of N-methylol nylon- 6 membranes for pervaporation of ethanol-water mixtures. J. Appl. Polym. Sci. 64, 855-863 (1997).

3 Chan, W. H., Ng, C. F., Lam-leung, S. Y., He, X. \& Cheung, O. C. Water-alcohol separation by pervaporation through poly(amide-sulfonamide)s (PASAs) membranes. J. Appl. Polym. Sci. 65, 1113-1119 (1997).
4 Chan, W. H., Ng, C. F., Lam-leung, S. Y., He, X. \& Cheung, O. C. Pervaporation of aqueous ethanol solution through poly(amidesulfonamide)s (PASAs) membranes. Polymer 39, 2461-2467 (1998).

$5 \mathrm{He}, \mathrm{X}$., Chan, W. H. \& Ng, C. F. Water-alcohol separation by pervaporation through zeolite-modified poly(amidesulfonamide). J. Appl. Polym. Sci. 82, 1323-1329 (2001).

6 Morgan, P. W. Condensation Polymers: By Interfacial and Solution Methods. (Interscience Publishers: New York, 1965), pp 1-561.

7 Rao, A. P., Joshi, S. V., Trivedi, J. J., Devmurari, C. V. \& Shah, V. J. Structureperformance correlation of polyamide thin film composite membranes: effect of coating conditions on film formation. J. Membr. Sci. 211, 13-24 (2003).

8 Veríssimo, S., Peinemann, K.- V. \& Bordado, J. Thin-film composite hollow fiber membranes: an optimized manufacturing method. J. Membr. Sci. 264, 48-55 (2005).

9 Li, L., Zhang, S., Zhang, X. \& Zheng, G. Polyamide thin film composite membranes prepared from 3,4',5-biphenyl triacyl chloride, 3,3',5,5'-biphenyl tetraacyl chloride and m-phenylenediamine. J. Membr. Sci. 289, 258-267 (2007).

10 Chen, G., Li, S., Zhang, X. \& Zhang, S. Novel thin-film composite membranes with improved water flux from sulfonated cardo poly(arylene ether sulfone) bearing pendant amino groups. J. Membr. Sci. 310, 102-109 (2008).

11 Liu, M., Wu, D., Yu, S. \& Gao, C. Influence of the polyacyl chloride structure on the reverse osmosis performance, surface properties and chlorine stability of the thin-film composite polyamide membranes. J. Membr. Sci. 326, 205 (2009).

12 Oh, N. W., Jegal, J. \& Lee, K. H. Preparation and characterization of nanofiltration composite membranes using polyacrylonitrile (PAN). II. Preparation and characterization of polyamide composite membranes. J. Appl. Polym. Sci. 80, 2729-2736 (2001).

13 Mohammad, A. W., Hilal, N. \& Seman1, M. N. A Interfacially polymerized nanofiltration membranes: atomic force microscopy and salt rejection studies. J. Appl. Polym. Sci. 96, 605-612 (2005)

14 Veríssimo, S., Peinemann, K.- V. \& Bordado, J. Influence of the diamine structure on the nanofiltration performance, surface morphology and surface charge of the composite polyamide membranes. J. Membr. Sci. 279, 266-275 (2006).

15 Buch, P. R., Mohan, D. J. \& Reddy, A. V. R. Preparation, characterization and chlorine stability of aromatic-cycloaliphatic polyamide thin film composite membranes. J. Membr. Sci. 309, 36-44 (2008).

16 Yoon, K., Hsiao, B. S. \& Chu, B. High flux nanofiltration membranes based on interfacially polymerized polyamide barrier layer on polyacrylonitrile nanofibrous scaffolds. J. Membr. Sci. 326, 484-492 (2009).

17 Huang, S. H., Li, C. L., Hu, C. C., Tsai, H. A., Lee, K. R. \& Lai, J. Y. Polyamide thin-film composite membranes prepared by interfacial polymerization for pervaporation separation. Desalination 200, 387-389 (2006).

18 Huang, S. H., Lin, W. L., Liaw, D. J., Li, C. L., Kao, S. T., Wang, D. M., Lee, K. R. \& Lai, J. Y. Characterization, transport and sorption properties of poly(thiol ester amide) thin-film composite pervaporation membranes. J. Membr. Sci. 322, 139-145 (2008).

19 Huang, S. H., Hung, W. S., Liaw, D. J., Li, C. L., Kao, S. T., Wang, D. M., Guzman, M. D., Hu, C. C., Jean, Y. C., Lee, K. R. \& Lai, J. Y. Investigation of multilayer pervaporation membrane by positron annihilation spectroscopy. Macromolecules 41, 6438-6443 (2008)

20 Li, Y., Zhang, R., Chen, H., Zhang, J., Suzuki, R., Ohdaira, T., Feldstein, M. M. \& Jean, Y. C. Depth profile of free volume in a mixture and copolymers of poly(N-vinylpyrrolidone) and poly(ethylene glycol) studied by positron annihilation spectroscopy. Biomacromolecules 4, 1856-1864 (2003).

$21 \mathrm{He}$, C., Hamada, E., Suzuki, T., Kumaki, T., Kobayashi, H., Kondo, K. \& Ito, Y. Pulsed slow-positron beam for polymer films. Appl. Surf. Sci. 221, 444-449 (2004).

22 Algers, J., Suzuki, R., Ohdaira, T. \& Maurer, F. H. J. Characterization of free volume and density gradients of polystyrene surfaces by low-energy positron lifetime measurements. Polymer 45, 4533-4539 (2004).

23 Jean, Y. C., Zhang, J., Chen, H., Li, Y. \& Liu, G. Positron annihilation spectroscopy for surface and interface studies in nanoscale polymeric films. Spectrochim. Acta. A. 61, 1683-1691 (2005).

24 Chen, H. M., Hung, W. S., Lo, C. H., Huang, S. H., Cheng, M. L., Liu, G., Lee, K. R., Lai, J. Y., Sun, Y. M., Hu, C. C., Suzuki, R., Ohdaira, T., Oshima, N. \& Jean, Y. C. Free-Volume depth profile of polymeric membranes studied by positron annihilation spectroscopy: layer structure from interfacial polymerization. Macromolecules 40, 7542-7557 (2007).

25 Huang, S. H., Hsu, C. J., Liaw, D. J., Hu, C. C., Lee, K. R. \& Lai, J. Y. Effect of chemical structures of amines on physicochemical properties of active layers and dehydration of isopropanol through interfacially polymerized thin-film composite membranes. J. Membr. Sci. 307, 73-81 (2008).

26 Li, C. L., Huang, S. H., Liaw, D. J., Lee, K. R. \& Lai, J. Y. Interfacial polymerized thinfilm composite membranes for pervaporation separation of aqueous isopropanol solution. Sep. Purif. Technol. 62, 694-701 (2008).

27 Oh, N. W., Jegal, J. \& Lee, K. H. Preparation and characterization of nanofiltration composite membranes using polyacrylonitrile (PAN). I. Preparation and modification of PAN supports. J. Appl. Polym. Sci. 80, 1854-1862 (2001).

28 Schut, H. \& van Veen, A. Software for modelling positron-beam depth-profiling data. J. Phys. IV 5, C1-57-C1-61 (1995).

29 Teng, M. Y., Lee, K. R., Fan, S. C., Liaw, D. J., Huang, J. \& Lai, J. Y. Development of aromatic polyamide membranes for pervaporation and vapor permeation. J. Membr. Sci. 164, 241-249 (2000).

30 Gao, Y., Robertson, G. P., Guiver, M. D., Jian, X., Mikhailenko, S. D., Wang, K. \& Kaliaguine, S. Sulfonation of poly(phthalazinones) with fuming sulfuric acid mixtures for proton exchange membrane materials. J. Membr. Sci. 227, 39-50 (2003). 